\title{
Cytomegalovirus Reactivation Finding
}

National Cancer Institute

\section{Source}

National Cancer Institute. Cytomegalovirus Reactivation Finding. NCI Thesaurus. Code C148545.

A laboratory test result indicating that a previously latent cytomegalovirus infection has become an active infection. 